\title{
NEOLIGNANAS DE UMA EMBUIA DO RIO GRANDE DO SUL, BRASIL
}

Francisco Frick Neto, Frida Maciel Pagliosa e Maria Elizabeth do Canto Vinadé

Departamento de Quimica. Centro de Ciências Naturais e Exatas. UFSM. Santa Maria, RS.

\section{RESUMO}

0 extrato hexānico do tronco de ocotea porosa, fracionado por tēcnicas cromatogräficas, forneceu trēs neolignanas do tipo ben zofurānico: porosina, (2R, 3S, 3aR, 5R)-3a-alil-3 metil-5 metoxi-2( $3^{\prime}$, $4^{\prime}$, dimetoxifenil)-6-oxo-2,3,3a,4,5,6-hexahidrobenzofurano; II re? (2R, 3S, 3a, 3aR, 5R, 5R, 6S, 7 a S)-3a-alil-6,7a-dihidroxi-3-metil-5 meto $x i 2-\left(3^{\prime}, 4^{\prime}\right.$-dimetoxifenil)-2-3-3a,4,5,6,7,7a-octahidrobenzofurano e III, rez (2R, 3S, 3aR, 5R,6S, 7 a S)-3a-alil-6,7a-dihidroxi-3 metil-5 metoxi-2-(3',4'-dimetoxifenil)-2,3,3a,4,5,6,7,7a-octahidrobenzofura no. As estruturas das neolignanas isoladas foram identificadas por anālise espectral no IR e de RMN de ${ }^{1} \mathrm{H}$ e por comparação com dados espectrais de compostos idēnticos.

\section{SUMMARY}

FRICK, F.N.; PAGLIOSA, F.M. and VINADE, M.E.C., 1988. Neolignanas from an Embuia of Rio Grande do Sul, Brazil. Ciência e Na tura, 10:89-98.

The $n$-hexane of the bark of ocotea porosa fractioned by chromatographic tecniques, afforded there neolignanas of benzofura noid, type: porosin, (2R,3a,3aR,5R)-3a-allyl-3-methyl-5-methoxy-2( $3^{\prime}, 4^{\prime}$, dimethoxypheny 1)-6-0x0-2,3,3a,4,5,6 hexahydrobenzofuran; II, rel (2R,3S, 3aR, 5R,6S, 7 a S)-3a-allyl-6-7a-dihydroxi-3-methyl-5-metho xy-2-( $3^{\prime}, 4^{\prime}$, dimethoxypheny 1$)-2-3-3 a, 4,5,6,7,7 a-o c t a h i d r o b e n z o f u r a n$, and II, rel (2R,3S,3aR,5R,6R,7 a S)-3a-allyl-6,7a-dihidroxy-3-methyl -5-methoxy-2-( $3^{\prime}, 4^{\prime}$, dimethoxypheny 1$)-2,3,3 a, 5,6,7,7$ a-octahydrobenzofu ran. The structures of isolated neolignanas were identified using IR, ${ }^{1} H$ spectral analysis and by comparison with published data of similar compounds.

INTRODUÇÃO

A famîlia Lauráceae na Rio Grande do Sul engloba nove gê neros com trinta espécies nativas.

ocotea porosa é uma das onze espēcies pertencentes ao gêne ro ocotea (22). A ocorrência da espécie no Rio Grande do Sul está na Figura 1 .

o estudo químico de mesmo specimen de outras regiões do Brasil jā foi desenvolvido anteriormente, do que resultou o isolamento 
de vārios tipos de substāncia, especialmente às pertencentes ao gru po das neolignanas $(1,2,3,9,11,17)$.

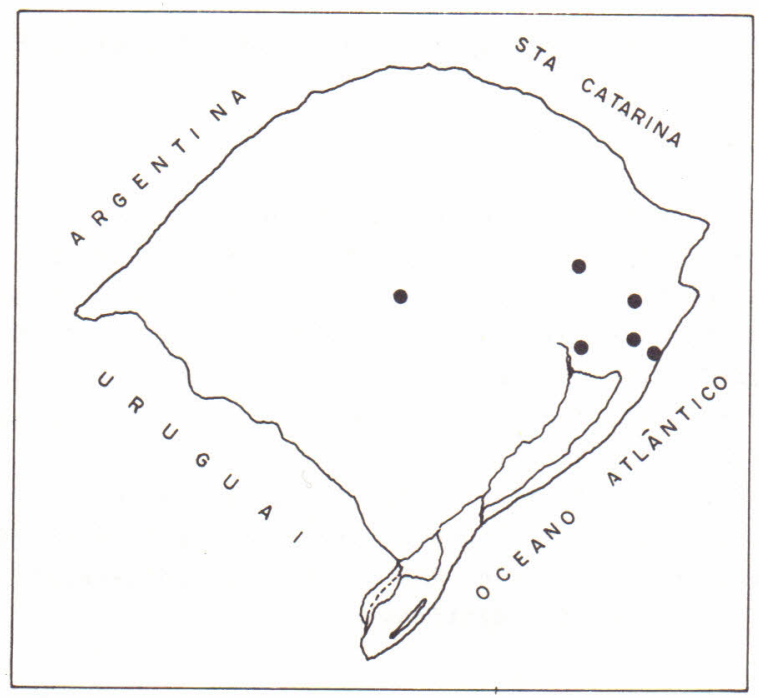

Figura 1 - Ocorrēncia de Ocotea porosa no Rio Grande do Sul.

As classes de neolignanas com maior número de representan tes foram as das neolignanas benzofurânicas e biciclootânicas. Fór mulas de algumas destas substâncias estão representadas a seguir.

FÓRMULAS DE ALGUMAS NEOLIGNANAS FARMACOLOGICAMENTE ATIVAS.<smiles>C[C@@H](Cc1ccc(O)c(O)c1)[C@H](C)Cc1ccc(O)c(O)c1</smiles>

Ácido nor-dihidroguaiarético

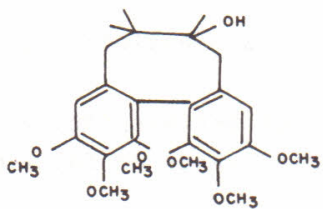

Esquizandrina

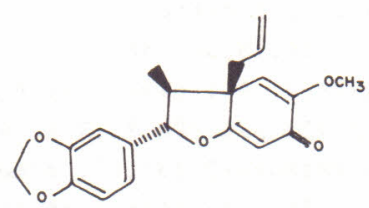

Burchelino<smiles>C/C=C/c1cc(OC)c2c(c1)[C@@H](C)[C@H](c1ccc(O)c(OC)c1)O2</smiles>

Licarina 


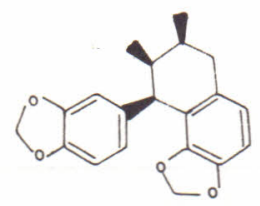

Otobaino<smiles>C=CCC1=C[C@]2(O)C(=CC1=O)O[C@H](c1ccc(OC)c(OC)c1)[C@@H]2O</smiles>

Kodsurenona

Algumas neolignanas apresentaram atividade biolōgica com provada $(14,20)$. Acido nor - dihidroguaiarētico $(22,24)$, Burchelina (5), esquizandrina (18), licarina (4), otobaina (16) são exemplos de neolignanas com propriedades anti-tumorais ou citotóxicas ou biodi nämicas. Kadsurenona, isolada recentemente de planta chinesa (8) com portou-se "in vitro" como potente inibidor da ação do PAF (Fator Ativante de Plaquetas) (19). O PAF ē responsāvel por certas enfermi dades como asma, hipertensão, anafilaxias cardíacas e artrites (6, 20). Mirandina-A com atividade menor, foi isolada de espécie amazo nense (21).

Estes fatos abrem novos horizontes quanto às aplicações me dicinais de neolignanas (especificamente benzofurânicas) das quais mais de 300 foram isoladas de espécies brasileiras.

Portanto, estes fatos tornaram a pesquisa da espécie gaú cha de grande interesse não só cientîfico como prático, porque cria bases para possỉveis aplicações práticas dos resultados e contribui para a formação quîmica de Produtos Naturais.

\section{MATERIAL E METODO}

0 material botānico em estudo, Ocotea porosa (Nees) J. An gely foi coletada na Estação Experimental de Silvicultura, próxima a Santa Maria (RS), região da Depressão Central. Está catalogada no Herbārio do Departamento de Biologia da Universidade Federal de San ta Maria sob no 2540.

ocotea porosa, conhecida popularmente como embuia, imbuia ou canela (23) è uma àrvore que atinge atē $25 \mathrm{~m}$ de altura possuindo caule com $45 \mathrm{~cm}$ de diâmetro e de odor resinoso e agradável. Após se cagem e moagem da casca do tronco, foi preparado o extrato hexānico. o fracionamento do extrato hexânico foi feito através de métodoscro matogräficos, conforme o Esquema 1 .

As frações obtidas por eluição em sistema de solvente he xano-benzeno, em proporção $8: 2$, após reunidas, foram submetidas à -recristalização em benzeno - hexano (9:1). As frações reunidas em decorrência da eluição em hexano-benzeno (1:1) e examinadas em cro matografia em camada delgada comparativa (CCDC), foram apōs submetị das à separação em cromatografia em camada delgada preparativa (CC DP). 


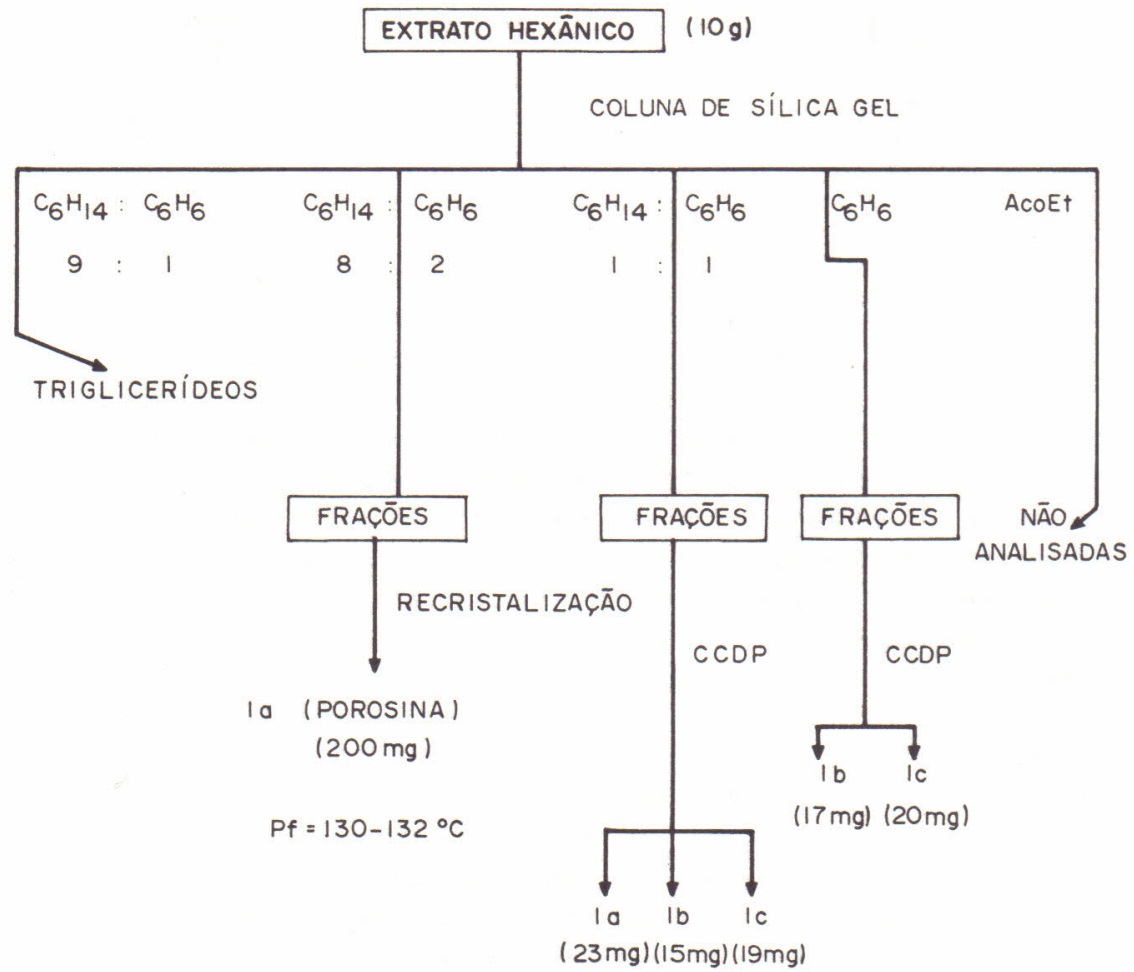

Esquema 1 - Fracionamento do extrato hexānico de Ocotea porosa.

As frações obtidas em benzeno, foram submetidas à CCDC em benzeno: acetato de etila (1:1).

As demais frações eluidas em benzeno: hexano (1:1) e ace tato de etila, não foram analisadas por se tratarem de misturas com plexas sem interesse imediato.

As estruturas isoladas ( $1 a, 1 b$ e $1 c)$ foram identificadas por anālise espectral de RMN de ${ }^{1} \mathrm{H}$ e no IR em comparação com dados de substâncias semelhantes na 1 iteratura $(1,2,3,9,11,17)$.

RESULTADOS E DISCUSSÃO

A substância la foi identificada como sendo a porosina, por comparação direta com amostra autêntica atravēs de cromatografia em camada delgada e com dados espectroscópicos descritos na literatura $(2,3)$. As substâncias 1 b e lc foram identificadas como sendo neo lignanas do tipo benzofurânicas por comparação com dados espectrais da literatura (9).

Os dados espectrais de RMN de ${ }^{1} H$ das substancias isoladas estão nas Tabelas I, II e III respectivamente.

Os espectros no IR estão na figura 2 . 
TABELA I - DADOS ESPECTRAIS DE RMN DE ${ }^{1} \mathrm{H}(60 \mathrm{MHz})$ DE la EM CDCl 3

\begin{tabular}{lllc}
\hline \hline PROTONS & M & \multicolumn{2}{c}{$1 \mathrm{a}$} \\
& & $\delta(\mathrm{ppm})$ & $\mathrm{J}(\mathrm{Hz})$ \\
\hline $\mathrm{H}-2$ & $\mathrm{~d}$ & 5,85 & 8,00 \\
$\mathrm{H}-3$ & $\mathrm{~m}$ & $2,50-2,80$ & - \\
$\mathrm{H}_{3} \mathrm{C}-3$ & $\mathrm{~d}$ & 0,55 & 8,00 \\
$2 \mathrm{H}-\alpha$ & $\mathrm{m}$ & $2,30-2,60$ & - \\
$\mathrm{H}-3$ & $\mathrm{~m}$ & $2,75-2,95$ & - \\
$2 \mathrm{H}-\mathrm{\gamma}$ & $\mathrm{m}$ & $5,30-5,60$ & - \\
$2 \mathrm{H}-4$ & $\mathrm{~m}$ & $1,85-200$ & - \\
$\mathrm{H}-5$ & $\mathrm{~m}$ & $3,90-4,20$ & - \\
$\mathrm{H}-7$ & $\mathrm{~s}$ & 5,55 & - \\
$\mathrm{H}_{3} \mathrm{CO}-5$ & $\mathrm{~s}$ & 3,60 & - \\
$2 \mathrm{H}_{3} \mathrm{CO}-\mathrm{Ar}$ & $\mathrm{s}$ & 3,88 & - \\
$3 \mathrm{H}-\mathrm{Ar}$ & $\mathrm{m}$ & $6,65-6,85$ & - \\
\hline
\end{tabular}

TABELA II - DADOS DE RMN DE ${ }^{1} H(60$ MHZ) DA LIGNANA Ib EM CDC1 3

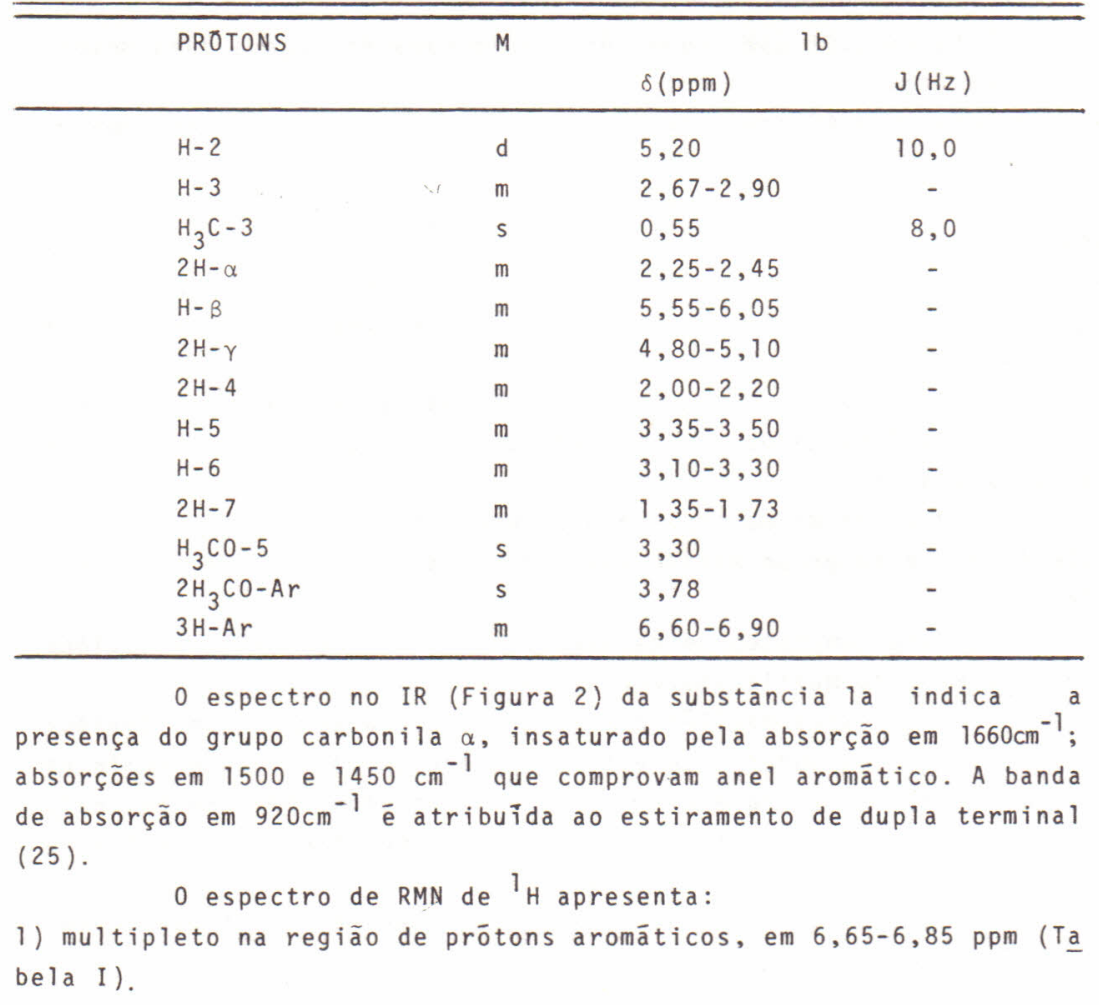


TABELA III - DADOS ESPECTRAIS DE RMN DE ${ }^{1} H(60 \mathrm{MHZ})$ DA LIGNANA IC EM $\mathrm{CDCl}_{3}$.

\begin{tabular}{|c|c|c|c|}
\hline \multirow[t]{2}{*}{ PROTONS } & \multirow[t]{2}{*}{ M } & \multicolumn{2}{|c|}{$1 \mathrm{c}$} \\
\hline & & $\delta(p p m)$ & $\mathrm{J}(\mathrm{Hz})$ \\
\hline $\mathrm{H}-2$ & d & 5,25 & 8,0 \\
\hline $\mathrm{H}-3$ & $\mathrm{~m}$ & $2,67-2,90$ & - \\
\hline $\mathrm{H}_{3} \mathrm{C}-3$ & $d$ & 0,55 & 8,0 \\
\hline $2 H-\alpha$ & $\mathrm{m}$ & $2,25-2,45$ & - \\
\hline$H-B$ & $\mathrm{~m}$ & $5,55-6,05$ & - \\
\hline $2 H-\gamma$ & $\mathrm{m}$ & $4,80-5,10$ & - \\
\hline $2 \mathrm{H}-4$ & $\mathrm{~m}$ & $1,87-2,17$ & - \\
\hline$H-5$ & $\mathrm{~m}$ & $2,90-3,18$ & - \\
\hline$H-6$ & $\mathrm{~m}$ & $3,65-3,91$ & - \\
\hline $2 \mathrm{H}-7$ & $\mathrm{~m}$ & $1,65-1,80$ & - \\
\hline $\mathrm{H}_{3} \mathrm{CO}-5$ & $\mathrm{~s}$ & 3,35 & - \\
\hline $2 \mathrm{H}_{3} \mathrm{CO}-\mathrm{Ar}$ & $s$ & 3,85 & - \\
\hline $3 \mathrm{H}-\mathrm{Ar}$ & $\mathrm{s}$ & 6,78 & - \\
\hline
\end{tabular}

2) singleto em $3,88 \mathrm{ppm}$, cuja integração para $6 \mathrm{H}$ sugere duas metox $\underline{j}$ las aromāticas.

3) singleto em $3,60 \mathrm{ppm}$, indicando por integração $3 \mathrm{H}$, sugere uma me toxila.

4) dubleto em $0,55 \mathrm{ppm}$ correspondente a $3 \mathrm{H}$, com J=8,00 Hz, indica 0 grupo metila de uma unidade $\mathrm{C}_{6} \mathrm{C}_{3}$.

$5)$ dubleto em $5,85 \mathrm{ppm}(\mathrm{J}=8,0 \mathrm{~Hz})$ è atribuīdo a próton oxibenzīlico.

6) multipleto em 5,30-5,60 ppm com 2H, sugere dupla terminal de um grupo alila.

7) singleto em 5,55 ppm sugere prōton ligado a carbono insaturado.

As informações apresentadas, são atribuídas a uma neoligna na benzofurānica.

0 valor do deslocamento químico da metila ligada a C-3, sugere que este grupo mantém com o grupo arila ligado em $\mathrm{C}-2$, rela ção cis.

Para compostos de estereoquímica relativa trans o desloca mento quimico da metila aparece em campo mais baixo $(7,10)$.

os prōtons metilênicos do grupo alila apresentam desloca mento químico que sugere que este grupo mantém com o grupo arila re lação do tipo trans. Para a relação cis os prōtons metilēnicos esta riam sob proteção do anel Benzēnico e consequentemente seu sinal de veria aparecer em campo mais alto (12).

As indicações acima relacionadas levaram à estrutura la, atribuỉda a neolignana porosina. 

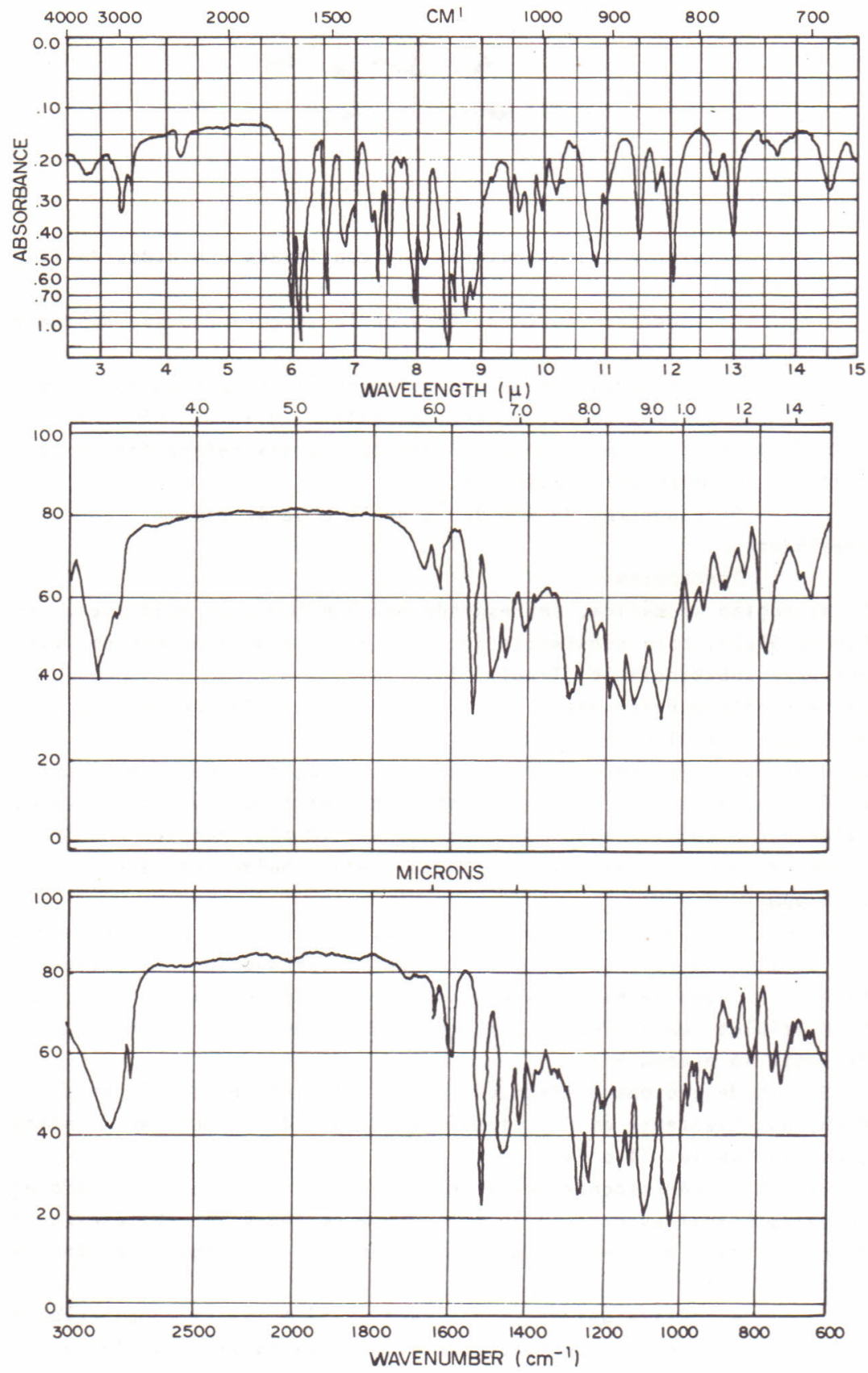

Figura 2 - Espectros no infravermelho de 1a, 1b e 1c. 


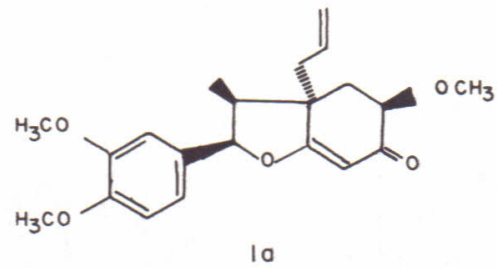

Esta proposta estrutural foi confirmada com dados da lite ratura $(1,2,6,9)$.

Os espectros no IR de lb e de lc (Figura 2), são bastante semelhantes.

Indicam presença de grupos hidroxilicos por bandas largas, em 3410 e $3430 \mathrm{~cm}^{-1}$; bandas de anel aromático em 1510, 1460, 1430 $\mathrm{cm}^{-1}$ e 1510,1490 e $1450 \mathrm{~cm}^{-1}$, respectivamente. Bandas referentes à pre sença de carbonila estão ausentes.

os espectros de RMN de ${ }^{1} H$ de lb e de lc tambēm, são muito semelhantes.

\section{Apresentam:}

1) $\mathrm{Na}$ região aromātica, correspondentea 3 prōtons, um multipleto em 6,60-6,90ppm, para substāncia 1b (Tabela II) e um singleto em 6,78 ppm para substāncia lc (Tabela II).

2) Um singleto referente a 6 prōtons de 2 metoxilas aromáticas, em $3,78 \mathrm{ppm}$ para II e 3,85 ppm para $1 \mathrm{c}$.

3) 0 grupo alila, em ambos os compostos é sugerido pelo multipleto em 5,55-6,05 ppm referente a próton $H-\beta$; multipleto em 2,25-2,45pm, referente a $2 \mathrm{H}-\alpha$; e multipleto em 4,80-5, $10 \mathrm{ppm}$, referente a $2 \mathrm{H}-\gamma$.

4) Um grupo metila alifático, $\left(\mathrm{CH}_{3}-3\right)$, ē atribuīdo pelo dubleto em $0,55 \mathrm{ppm}(\mathrm{J}=8,0 \mathrm{~Hz})$.

5) Singleto em $3,30 \mathrm{ppm}$, no espectro de $1 \mathrm{~b}$ e em $3,35 \mathrm{ppm}$, no espec tro de $1 c$, sugere grupo metoxila ligado a carbono $\mathrm{sp}^{3}$, provavelmen te em $\mathrm{C}-5$, como apresenta a estrutura $1 \mathrm{a}$, da porosina.

6) Multipleto em 2,67-2,90 ppm, referente a um próton alifático, é proposto ao próton $\mathrm{H}-3$.

7) Sinais de prōtons alifāticos, $2 \mathrm{H}$ em $\mathrm{C}-4$ (semelhante à porosina) e $2 \mathrm{H}$, possivelmente em $\mathrm{C}-7$, tambēm são sugeridos, conforme indica ções nas Tabelas II e III.

8) Os sinais correspondentes a $2 \mathrm{H}$ carbinōlicos, um $\mathrm{H}(\mathrm{H}-2 \mathrm{a})$ recober to pelas metoxilas entre 3,30 e 3,80 ppm em ambas as substāncias e outro indicado por $\mathrm{H}-6$ por multipleto em $3,10-3,30 \mathrm{ppm}$ para $1 \mathrm{~b}$ e $3,65-3,91$ para 1c.

A anālise dos dados das Tabelas II e II em comparaçãocom os dados da Tabela I, permite concluir que as substâncias lb e lc apresentam estruturas semelhantes às da porosina e que a diferença entre lb e 1c, estaria na orientação relativa dos grupos do anel 
ciclohexānico.

Anālise mais detalhada nos valores dos sinais protōnicos, dos espectros de RMN de ${ }^{1} H$ de $1 b$ e $1 c$, sugere que a diferença mais significativa referem aos valores de $\mathrm{H}-6$.

Este prōton, em lb, absorve em campo mais baixo que em 1c. Este fato poderia sugerir que $\mathrm{H}-6$ de 1 b apresenta uma orientação axial e portanto a hidroxila em C-6, ocuparia posição equatorial.

Estas conclusões propostas em relação as apresentadas em literatura (9) levam à proposição de estruturas para as substâncias, lb e lc, conforme formulação abaixo:

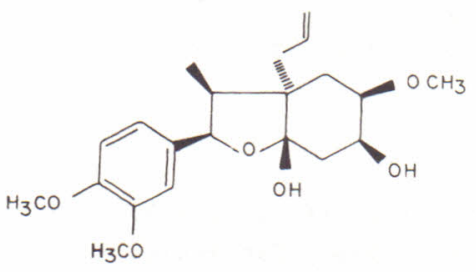

Ib

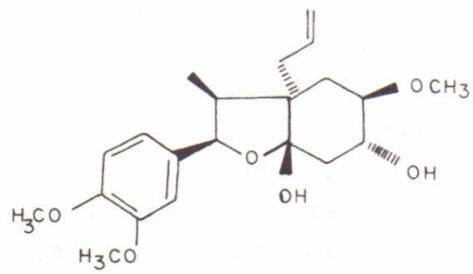

I c

CONCLUSOES

0 extrato $n$-hexânico da casca de ocotea porosa, coletada no Rio Grande do Sul, contēm la, lb e lc como constituintes quỉmicos em maior concentração.

As substâncias la, lb e lc são idênticas por comparação es pectral de RMN de ${ }^{1} \mathrm{H}$ e no IR com substāncias pertencentes a classe de neoligananas benzofurānicas isoladas de mesma espécimen, mas co letadas em outras regiões do Brasil.

0 estudo de Ocotea porosa nativa do Rio Grande do Sul, con tribui para complementar o estudo quỉmico de Lauráceas brasileiras.

\section{REFERENCIAS BIBLIOGRAFFICAS}

1. AIBA, C.J. Porosina, uma neolignana da imbuia. Instituto de Qui mica de São Paulo, USP, 1973. 120p. Tese Doutor. Quỉmica Orgâa nica.

2. AIBA, C.J.; BRAZ, R.FO and GOTTLIEB, O.R. (Porosin: a Neolignan from ocotea porosa). Phytochemistry, Great Britain, 12:143 $16,1973$.

3. AIBA, C.J.; GOTTLIEB, O.R.; YOSFIDA, M.; MOURAO, J.C. and GOTTLIEB, H.E. (The structure of Porosin. Phytochemistry, Great Britain, $15: 1031,1976$

4. AIBA, C.J.; CORREA, R.G.C. and GOTTLIEB, 0.R. Phytochemistry, Great Britain, 12:1163, 1973.

5. AlVARENGA, M.A. de; BROKSON, U.; GOTtLiEB, O.R.; YOSHIDA, M. J. Chem. Soc. Chem. Commun. $831,1978$. 
6. BENVENISTE, J.; BOULLET, C.; BRINK, C. and LABAT, C. Br. J. Pharmac., $80: 81,1983$.

7. CASTRO, C.0.; AlVARENGA, M.A.; GieSBRECHT, A.M. e GOTTLiEB, 0 . R. Phytochemistry, Great Britain, 16:1801, 1977.

8. CHANG, M.N. et al. Phytochemistry, Great Britain, 24:2079-2082, 1985.

9. DIAS, D.A. Neolignanas de Ocotea porosa. São Paulo, USP, 1985. 107p. Tese Doutor. Quỉmica Orgânica.

10. FERnANDES, J.B.; GOTTLIEB, 0.R.; AIBA, C.J. e MAIA, J.G.S. Phy tochemistry, Great Britain, 14:1597, 1975.

11. GOMES, P.C.M.C. Novas neolignanas biciclo [3.2.1] octânicas de uma espécie de ocotea. São Paulo, USP, 1981. 84p. Diss. Mes trado Quỉmica Orgānica.

12. GOTTLIEB, 0.R. Lignans and Neolignans. Rev. Latinoamer. Quim., $5: 1,1974$.

13. GOTTLIEB, 0.R. Phytochemistry, Great Britain, 11:1537, 1972 .

14. GotTlieb, 0.R. e YoShIDA, M. Quimica Nova, São Paulo, $7(4): 250$ 1984.

15. GOTTLIEB, 0.R. Progressing in the chemistry of Organic Natural Products. Viena, Pringer Verlag. V.35, 1978, 72p.

16. GIL CHRIST, T.; HODGES, R. and PORTE, A.L. t. Chem. Soc., London, $1780,1962$.

17. HARAGUCHI, M. Neolignanas de Ocotea catharinensis, São Paulo, USP, 1982. 11p. Diss. Mestr. Quîmica Orgânica.

18. KOTCHETKOV, N.K.; KHORLIN, A.Ya.; CHIZHO, 0.S. Chem. Abstr. 57: $3334,1962$.

19. LEVI, R. et al, Cire. Res. 54:117, 1984.

20. MACRAE, W.D. and TOWERS, G.H.N. Phytochemistry, Great Britain, $21(6): 1207,1984$.

21. PAGLIOSA, F.M.; GOTTLIEB, 0.R.; YOSHIDA, M. Phytochemistry Great Britain, 16:745-8, 1977 .

22. PARDINI, R.S.; HIDCKER, J.C. and FLETCHER, D.C. Biochem. Pharm. $19: 2695,1970$.

23. PEDRALLI, G. A famizia Lauraceae Lindiey no Rio Grande do Sul, Brasiz. Porto Alegre, UFRGS, 1981. 177p. Diss. Mestr. Botān $\mathrm{ca}$.

24. RUTH, E.F. Anales. Assoc. Quim. Argentina, 34:163, 1946.

25. SILVERSTEIN, R.M. Spectrometric identification of organic com pounds, 3 ed., New York, John Wiley, 1974.

Recebido em dezembro, 1988; aceito em dezembro, 1988. 